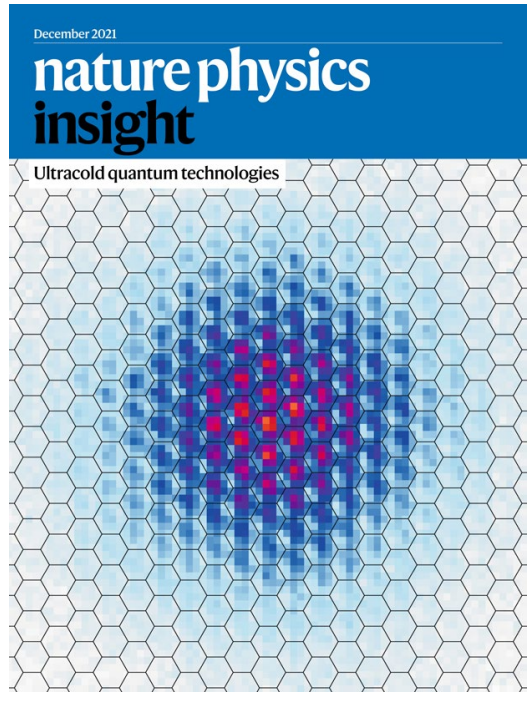

Image: Christof Weitenberg et al. Cover Design: Allen Beattie

\section{SPRINGER NATURE LONDON}

The Campus, 4 Crinan Street,

London N19XW

$\mathrm{T}:+442078334000$

F: +442078434563

nature.physics@springernature.com

CHIEF EDITOR

ANDREA TARONI

INSIGHT EDITORS

RICHARD BRIERLEY, YUN LI,

LEONARDO BENINI

PRODUCTIONEDITOR

FRANCESCA SANDFORD

SENIOR COPY EDITOR

KEVIN SHERIDAN

ART EDITOR

ALLEN BEATTIE

EDITORIAL ASSISTANT

JAMES TATAM

EDITORIAL DIRECTOR

ALISON WRIGHT

EDITOR-IN-CHIEF,

NATURE JOURNALS

MAGDALENA SKIPPER

\title{
Ultracold quantum technologies
}

$\mathrm{T}$

he creation of a Bose-Einstein condensate was a major milestone in the field of ultracold gases. Over seventy years passed from the first ideas predicting this phase of matter, which is characterized by a macroscopic occupation of a single quantum state, to its eventual experimental observation in 1995. But this was more than the confirmation of a long-standing theoretical prediction. Since this achievement, a rich toolbox provided by ultracold gases has become central to the study of quantum many-body physics. In this Insight we present an overview of some of the latest experimental advances that are pushing the field still further, expanding its influence across physics.

As discussed in the Comment by Jook Walraven, the achievement of Bose-Einstein condensation required a series of breakthroughs in the development of trapping, cooling and detection techniques. The rest of the collection is made up of Review Articles that show how fast the pace of progress has been. The fundamental methods needed to cool and probe ultracold gases have continued to improve, and new approaches to trapping and control have emerged. This suite of ultracold quantum technologies has reinforced the position of ultracold gases as the pre-eminent platform for studying collective quantum phenomena, while extending capabilities at the single-atom level.

It is not possible to do justice to a field as broad as ultracold gases in a single issue. In particular, there have been significant advances in engineering practical devices for applications including metrology and quantum computing, with commercial interest rapidly growing. We are sure that the collection of experimental technologies reviewed here will play a key role in these developments and in many fundamental and applied breakthroughs that are yet to come.

Richard Brierley, Senior Editor

Yun Li, Senior Editor Leonardo Benini, Associate Editor

Published online: 7 December 2021

https://doi.org/10.1038/s41567-021-01461-3

\section{COMMENT}

Cold atoms stay cool

Jook Walraven

p1294

REVIEW ARTICLES

Laser cooling for quantum gases

Florian Schreck and Klaasjan van Druten

p1296

Spectroscopic probes of quantum gases

Chris J. Vale and Martin Zwierlein

p1305

Quantum gas microscopy for single atom and spin detection

Christian Gross and Waseem S. Bakr

p1316

Quantum science with optical tweezer arrays of ultracold atoms and molecules

Adam M. Kaufman and Kang-Kuen Ni

Quantum gases in optical boxes

Nir Navon, Robert P. Smith and Zoran Hadzibabic

Tailoring quantum gases by Floquet engineering Christof Weitenberg and Juliette Simonet

Developments in atomic control using ultracold magnetic lanthanides

Matthew A. Norcia and Francesca Ferlaino 